graphs taken by Mr. Fowler during the eclipse of 1893 (Phil. Trans., A, vol. I 87, p. 593). Among the brightest of these rings, which is common to all three sets of photographs, is one about wave-length $423 \mathrm{I}$, which probably is identical with the corona line photographed by Schuster in 1886 , and stated to have a wave-length of $4^{232 \cdot 8}$ on Angström's scale (4233.4 Rowland). Schuster stated that this line was "probably the same line as $4233^{\circ} \mathrm{O}$ often observed by Young in the chromosphere" (Phil. Trans, , A, vol. 180, p. 34I). The chromospheric.line at this wave-length has since been identified as an enhanced line of iron, of which the precise wave-length is 4233.3. Captain Hills photographed this corona line with a slit spectroscope in the last eclipse, and he gives its wavelength as $4233^{\circ} 5$ (Roy. Soc. Proc., vol. 64, p. 54), which within the limits of error might be considered coincident with the enhanced line of iron.

The later researches on the spectrum of iron have shown that the iron line which I observed in 1869 to be coincident with the bright chromospheric line at $1474 \mathrm{~K} \quad\left(5316^{\circ} 79\right.$ Rowland) is also an enhanced line, agreeing absolutely with Young's latest determination of the wave-length of the 1474 chromospheric line (Scheiner's "Astronomical Spectroscopy,"
With regard to the ring in the green, the lack of sufficient photographs on isochromatic plates in 1893 does not permit of a final determination of wave-length. Important data, however, were obtained, both in 1896 and 1898 . A measurement of the position of the chief ring in the green, as shown in these photographs, comparing the ring with the spectrum of the chromosphere and a solar and iron spectrum taken by the same prisms, shows beyond all question that the wave-length is very different from that generally accepted. The mean result of measurements of different parts of the ring made by Messrs. Fowler and Shackleton and Dr. Lockyer is $5303 \%$, or about I3 tenth-metres more refrangible than $1474 \mathrm{~K}\left(53^{1} 6^{\circ} 79\right)$.

Although the new wave-length is not to be regarded as final, for the reason that the conditions under which the photographs were taken necessitate certain small corrections which have not yet been fully worked out, it is not likely that it can be in error by so much as $I$ tenth-metre.

The examination of the photographs, which has been undertaken in the first instance by Mr. Fowler, indicates that other important conclusions are to be drawn from the admirable series obtained by him, among them the possible existence of one or more new gases, some of the lines of which, as gathered from the

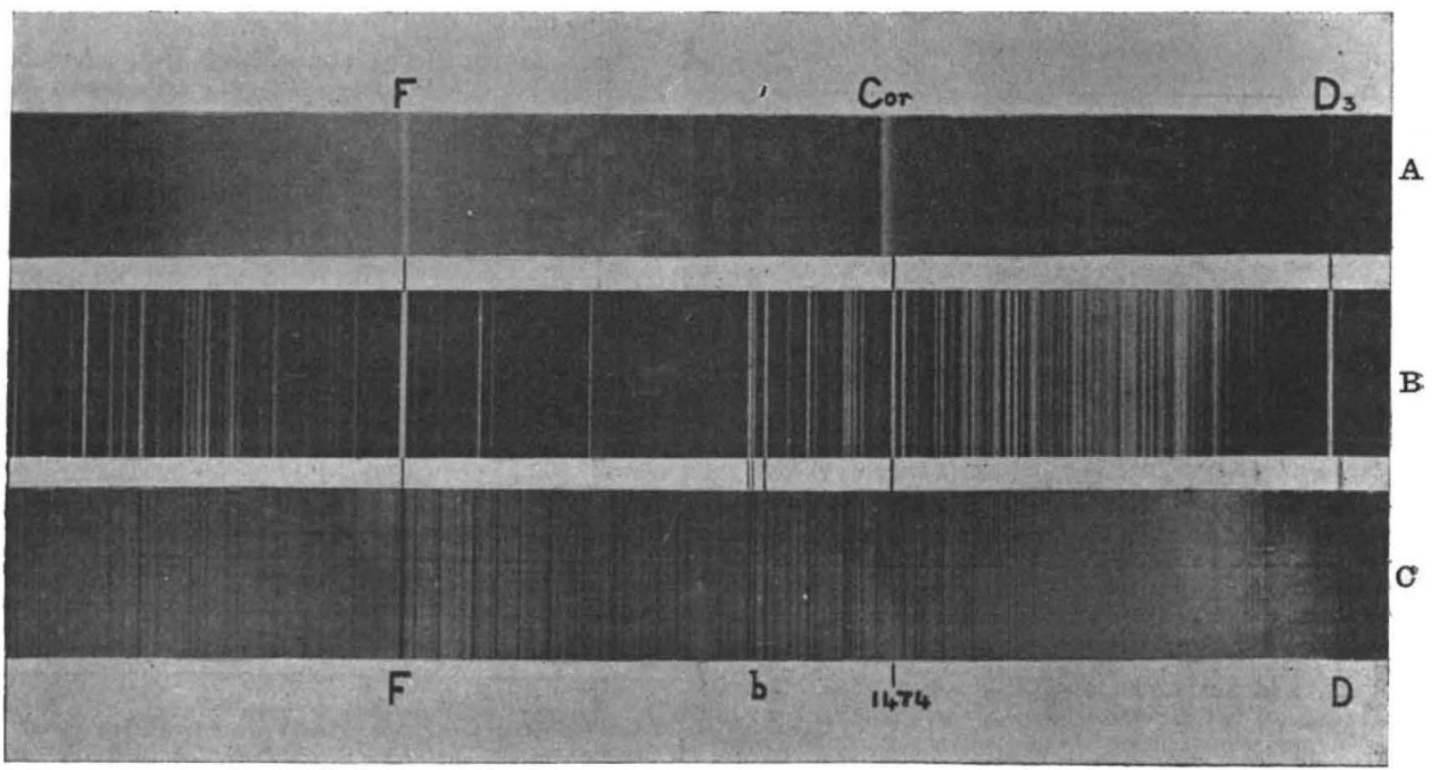

Comparison of the position of the chief line in the spectrum of the corona (A) with the enhanced line of iron at $\mathrm{r}_{474} \mathrm{~K}$, seen in the spectrum of the chromosphere (B) and in the ordinary solar spectrum C.

Frost's translation, p. 425), with which, according to his eclipse observations, the green line of the corona is coincident.

According to these results then, two of the chief lines in the spectrum of the corona would be coincident with enhanced lines of iron. The remaining corona lines which have so far been measured, are not, however, coincident with enhanced tines. It did not seem possible, therefore, that two of the enhanced lines of iron should be present without the others, even if it be admitted that the corona may have a tempe rature high enough to produce any enhanced lines.

It appeared then, either that the coincidences of the chromospheric and coronal lines about 423 and 531 were accidental, or that they were not real coincidences at all. A careful examination of the eclipse photographs of I896, taken by $\mathrm{Mr}$. Shackleton, and those of 1898 , taken by Mr. Fowler, has therefore been undertaken, with special reference to this point.

The wave-length of the coronal ring at $423 I$, already published in case of the 1893 photographs, has been confirmed.

The 1896 and 1898 photographs further indicate that the corona line near $423 \mathrm{I}$ is not coincident with the chronıspheric line to which reference has been made, and show that while the chromospheric line is coincident with the enhanced line of iron at $\lambda 4^{2} 33^{\circ} 3$, the corona line has a wave-length of $423 I^{\circ} 3$.

NO. I 525 , vOL. 59] dispersions as yet available, appearing also in the spectra of some stars and planetary nebulæ.

The photograph which accompanies this paper has been prepared by Mr. Fowler.

\section{HIGH VACUA PRODUCED BY LIQUID HYDROGEN.1}

$A \mathrm{~S}$ an illustration of the extraordinary power of the new cooling agen --liquid hydrogen, the extreme rapidity with which high vacuo can be produced by its use is, perhaps, one of the most striking. The absolute boiling points of hydrogen, oxygen, and chlorine are respectively $35^{\circ}, 90^{\circ}$ and $240^{\circ}$, in other words oxygen boils at a temperature two and a half times higher than liquid hydrogen, and liquid chlorine similarly at two and a half times that of liquid oxygen. From this we infer that liquid hydrogen as a cooling agent ought to be relative to liquid air as effective as the latter is compared to that of liquid chlorine. Now chlorine at the temperature of boiling oxygen is a hard solid, some $80^{\circ}$ below its melting point, and in this condition has an excessively feeble vapour pressure.

1 "Application of Liquid Hydrogen to the Production of High Vacua together with their Spectroscopic Examination." Paper read at the Royal Society on December 15, 1898, by James Dewar, F.R.S. 
When liquid hydrogen freezes air out of a sealed tube by immersing the end in the liquid, it is to be inferred that no measurable pressure of air ought to be left in the vessel. If we apply Van der Waals's law of corresponding temperatures to the case of hydrogen, the above inference is made unimpeachable. An approach to some knowledge of what the tension of air must be about the boiling point of hydrogen can be attained by exterpolating the vapour pressure curves of oxygen and nitrogen. Taking the following range of boiling point temperatures for nitrogen and oxygen, viz. from the critical point to the boiling point under diminished pressure, two Willard Gibbs formulæ were calculated, with the following results :-

$$
\begin{aligned}
& \text { Nitrogen } \ldots\left\{\begin{array}{lllll}
\text { Temp. abs. } \ldots . . . . . & 127^{\circ} & 78 \cdot 6^{\circ} & 59^{\circ} \\
\text { Pressure in mm. } \ldots & 25,900 & 740 & 26
\end{array}\right. \\
& \text { Nitrogen. } \log _{10} p=\mathrm{Ir} \cdot 556 \mathrm{r}-\frac{400 \cdot 02}{\mathrm{~T}}-\mathrm{r} \cdot 8980 \log _{10} \mathrm{~T} \ldots(\mathrm{I}) \text {. } \\
& \text { Oxygen } \ldots\left\{\begin{array}{lccr}
\text { Temp. abs......... } & 154^{\circ} & 90 \cdot 3^{\circ} & 61 \cdot 3^{\circ} \\
\text { Pressure in mm... } & 37,592 & 740^{\circ} & 7 \cdot 5
\end{array}\right. \\
& \text { Oxygen. ... } \log _{10} p=9.4699-\frac{422 \cdot 22}{\mathrm{~T}}-0.9843 \log _{10} \mathrm{~T} . .(2) \text {. }
\end{aligned}
$$

Another Gibbs formula was calculated, taking Estreicher's values for the vapour pressure of liquid oxygen below its boiling point, viz. :-

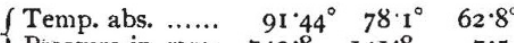

$$
\begin{aligned}
& \text { Pressure in } \mathrm{mm}: \quad 743.8 \text { I4I.8 } 7.5
\end{aligned}
$$

$$
\text { Oxygen. } \log _{10} p=16.0670-\frac{524 \cdot 72}{\mathrm{~T}}-3.8024 \log _{10} \mathrm{~T} \text {. . (3). }
$$

We deduce from these formulæ the following vapour pressures at the temperature of boiling hydrogen :-
(I) Nitrogen $\quad \ldots \quad 0.0015$
(2) Oxygen $\quad \ldots \quad 0.000076$
Pressure in mm., $35^{\circ}$ abs.
(3) $", \quad \ldots 0000016$
do.
do.

The results of calculation, taking the formula for the widest range of pressures, viz. (I) and (2), may probably be the surest, but in any case those values must be taken as a maximum, seeing they refer to the liquid state, while both oxygen and nitrogen, at the temperature of $35^{\circ}$ absolute, are hard solids, and must therefore have dropped to lower tensions than that of the exterpolated liquid vapour pressure curves. It is curious to note that at this low temperature the theoretical ratio of the tensions of nitrogen and oxygen is as 20 to $\mathrm{r}$. Direct measure ments of the vapour pressure of nitrogen at the melting point, or $60^{\circ}$ absolute, gave the value of $26 \mathrm{~mm}$., and a ratio of the tensions of nitrogen to oxygen of 6 to 1 , whereas from the curves the value ought to be 6.7 to $\mathrm{I}$. Olszewski gives the tension of nitrogen at $-214^{\circ}$ as $60 \mathrm{~mm}$., and as at this temperature the oxygen tension is $3.8 \mathrm{~mm}$., the ratio of the saturated pressures of the two gases at the melting point of nitrogen would be as 16 to $\mathrm{I}$, which is far too high. Probably the oxygen value will be nearest the truth, seeing it has the lowest melting point. The tension is about a ten millionth of an atmosphere. In the case of nitrogen, the maximum theoretical pressure would be one five hundred-thousandth of an atmosphere. It is safe to infer that the vacuum left after liquefying the air out of a vessel by means of liquid hydrogen cannot exceed the millionth part of the atmospheric pressure, exclusive of the pressure resulting from any incondensable material other than nitrogen and oxygen. This is just about the pressure of the vapour of mercury at the ordinary temperature in the Torricellian vacuum, so that as good an exhaustion ought to result as can be got by boiling out a space with mercury. There is another way in which the question may be put. Assuming the molecular latent heats are approximately proportioned to the absolute boiling points, then we can, from a comparison with the oxygen value, deduce that of hydrogen, and thereby get the constants in a two term formula for the vapour pressures. For pressures below an atmosphere, the following approximate formulæ were deduced:-

$$
\begin{aligned}
& \text { Oxygen } \ldots \ldots \log p=7 \cdot 2058-\frac{392 \cdot 6}{\mathrm{~T}} \mathrm{~mm} . \quad .(4) . \\
& \text { Hydrogen } \ldots \log p=7 \cdot 2428-\frac{152 \cdot 7}{\mathrm{~T}} \mathrm{~mm} . \quad .(5) .
\end{aligned}
$$

NO. I 525 , vOL. 59]
From these expressions it follows that at its boiling point, or $35^{\circ}$ absolute, hydrogen has 7/852000 times the pressure of oxygen, or the latter pressure is about the eight millionth of an atmosphere. A similar formula, calculated from the critical and boiling point data, gives substantially the same order of quantities. Formulæ (4) for oxygen tensions must be fairly accurate, seeing it gives a theoretical latent heat of about $5^{6}$ units per gram of liquid evaporating at the boiling point, whereas direct determinations result in 55 units. To test this inference, the following plan of experimenting was adopted:-Ordinary shaped vacuum tubes, like A, B, used for the spectroscopic examination of gases, with and without electrodes (Figs. I and 2), having a capacity ranging from 15 to 25 c.c., had pieces of quill tubing about a foot long sealed on. The tubes were contracted at $\mathrm{D}$ to about I mm., so that they could be sealed off with rapidity. The end $c$ sometimes terminated in a small bulb (Fig. 3), in order to give increased cooling surface, and, when necessary, to allow many times the volume of air in A, B, to enter and be condensed with the object of accumulating any incondensable residuum.

The tubes were filled with air, oxygen, and nitrogen at the atmospheric pressure. The liquid hydrogen collected in the vacuum vessel, immersed in another similar vessel full of liquid air, being ready, the end $\mathrm{C}$ was dipped in the liquid for a little over a minute, and the tube $A B$ sealed off at $D$, so that on removal from the hydrogen bath the solid air might melt and distil off without generating any pressure. On attempting to

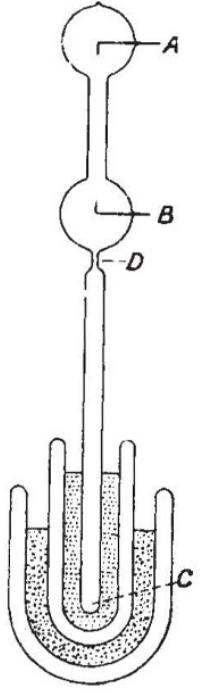

FIG. I.

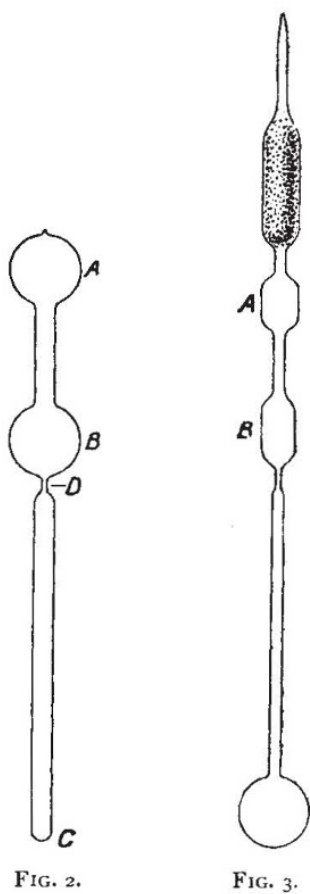

FIG. 2. pass the spark through vacuum tubes prepared in this manner, their excellent exhaustion was revealed by great resistance to the passage of the discharge, and the high phosphorescence of the glass. Two tubes, kindly prepared by Sir William Crookes with platinum electrodes that he had previously sparked to remove gases and impurities on the glass before filling with dry air, gave, when treated in the manner described, such high vacua that the tubes had to be heated in order to get any spark to pass. Thus it is proved that the tension of solid nitrogen and oxygen at the temperature of boiling hydrogen is below the millionth of an atmosphere, seeing there is less difficulty in getting a discharge to pass in tubes exhausted to this extent. In order to get some definite idea of the limit of the exhaustion produced, two tubes, such as have been described as suitable for the liquid hydrogen experiments, might be joined together and filled with oxygen or nitrogen at atmospheric pressure, and simultaneously exhausted with the mercurial pump to a small fraction of an atmosphere, and then sealed off from the pump 
and each other. One of these two identical tubes could then be subjected to the hydrogen cooling, following the directions already given, and the two vacuum tubes now compared. If there was a marked difference in resistance to the passage of the discharge in the frozen tube, then something must have condensed, and by a few tentative trials a linit might be reached when the initial exhaustion was unaffected by the hydrogen cooling. Such experiments have not yet been made. The presence of any vapour of mercury would require to be carefully eliminated, otherwise the method would not be satisfactory. Tubes that are prepared without taking special precautions to exclude organic matter and water from the glass, deteriorate, especially with electrodeless tubes after the discharge has taken place for some time.

The rapidity with which the vacua are attained is such as theory would suggest, assuming a hole of a square millimetre in section through which the air rushes into the condenser and that a velocity of current between 600 and 700 feet a second is attained, then a vessel of 20 c.c. capacity could be reduced in pressure in I second to I/IO of the initial pressure, and if the same rate is continued at the end of 60 seconds to $\left(\frac{1}{10}\right)^{60}$. Sir George Stokes has been good enough to consider the problem and writes as follows:-

"Let V be the volume of the vessel, A the area of an aperture by which the air is conceived as rushing out with the velocity $v, \rho$ the density of the air in the vessel at the time $t$, $\mathrm{D}$ the initial density, that is, the atmospheric density.

"Then, according to our hypothesis, Av.dt is the volume of air, and, therefore, Avp.dt, the mass of air, which rushes out in the time $d t$. But this equals the loss of mass of air in the vessel during the time $d t$, and, therefore,

$$
\mathrm{A} v \rho \cdot d t=-\mathrm{V} d \rho,
$$

a differential equation of which the integral is

$$
\rho=\mathrm{D} e^{-\mathrm{A} v t / \mathrm{V}} \text {. }
$$

"Suppose now V to be 20 c.c., or $20,000 \mathrm{c.mm}$., A to be the area of a circle of I or $2 \mathrm{~mm}$. diameter, say $2 \mathrm{sq}$. $\mathrm{mm}$., $v$ to be $333 \mathrm{~m}$., or $333,000 \mathrm{~mm}$., $t$ (in seconds) to be 60 ; then

$$
\begin{gathered}
\log _{e} \frac{\mathrm{D}}{\rho}=\frac{2 \times 3.33,000 \times 60}{20,000}=1998, \\
\frac{\mathrm{D}}{\rho}=5254 \times 10^{434} .
\end{gathered}
$$

"This would give a density of almost inconceivable smallness. Doubtless the supposition made above as to the rate of discharge is very wide of the mark, being much too great. If the velocity of rushing is about half the velocity of sound, the ratio of densities would become $72 \times 10^{217}$. If so it is satisfactory to find that the mathematical following out of the hypothesis leads to a density of the residual air in the vessel which is enormously below what suffices to account for the observed result." A practical mode of rapidly attaining a high vacuum in any vessel is to displace the air with carbonic or sulphurous acid, either at the atmospheric or under diminished pressure, and then to freeze out the remaining gas by the use of liquid air, just as in the experiments with liquid hydrogen.

The first vacuum tube was an electrodeless one, the air had not been dried, nor the glass specially cleaned. On spectroscopic examination it showed hydrogen lines bright along with the second or compound line spectrum of the same gas, and a series of bright bands defined on the less refrangible side, diffuse on the more refrangible, which occur in the yellow, green, blue, and indigo. These bands were found to be identical with the carbonic oxide spectrum. With a Leyden jar in the secondary circuit the line spectrum of hydrogen disappeared, leaving the second spectrum fainter; but the carbonic oxide bands remained bright, and there was no appearance of the hydrocarbon spectrum. The second tube had aluminium electrodes, and, like the last, had no special treatment in filling in the air. This tube showed also the line spectrum and the second spectrum of hydrogen; the latter being bright along with the carbonic oxide spectrum; but on sparking the latter disappeared. No appearance of the hydrocarbon spectrum could be detected, but there was a suspicion of bands in the indigo like the negative pole spectrum of nitrogen. The addition of a Leyden jar brought out nothing new, only intensifying the line

$$
\text { NO. I } 525 \text {, vOL. 59] }
$$

spectrum of hydrogen, while leaving the second spectrum bright. In neither of the above tubes could any lines of nitrogen or oxygen be recognised. The third tube was filled with air drawn over cotton wool, red-hot copper oxide, and phosphoric pentoxide, no rubber joints being employed. The spectrum showed the carbonic oxide bands and the hydrogen line spectrum as before. Only the second hydrogen spectrum was feeble. There was a yellow line W.L. 5849 , identical with one occurring in the natural gas from the King's Well at Bath. In a paper on " The Liquefaction of Air and the Detection of Impurities" (Chem. Soc. Proc., November 1897), the separation of helium from this gas is described by liquefaction and fractionation, and it was observed that during the sparking the helium lines were well marked along with "others, the origin of which must be settled laler." It was further observed, "With a modified form of apparatus it will be possible to collect any residuary gas from the use not of 3 cubic feet of air or Bath gas, but from hundreds of cubic feet of such products." The helium and other associated material was shown to be more volatile than nitrogen. Pursuing this course of investigation in the summer of this year, the volatile portion of air was examined, when the presence of material giving the same lines as Bath helium was recognised. While this investigation was in progress, Prof Ramsay and Dr. Travers observed the same spectrum in the more volatile portion of argon which they have associated with a new element called neon. The use of liquid hydrogen, as described, proves that the most characteristic line of neon in the yellow, about W. L. 5849, can be detected in 25 c.c. of ordinary air, and the presence of helium in the atmosphere is confirmed. ${ }^{1}$

A fourth tube, filled like the preceding one, had a phosphoric pentoxide tube left on. This showed again the carbonic oxide bands, but no hydrogen lines could be detected; while the oxide of copper ought to have removed all free hydrogen and transformed all the organic matter into carbonic acid and water. Yet it appears that the spectrum of the carbon compounds is difficult to remove from electrodeless tubes, probably owing to carbonic acid coming from the glass. There were some broad diffuse bands that may arise from the drying agent. The absence of hydrogen in this tube suggests that its presence in the third tube was due to vapour of water coming slowly from the glass. I am greatly indebted to Prof. Liveing for making a careful examination of the spectra of these tubes.

Sir William Crookes was good enough to prepare two tubes with platinum electrodes, which he sparked in vacua till all hydrogen disappeared, and then filled with dry air, but without the use of red-hot copper oxide or any agent for the absorption of carbonic acid or the destruction of organic matter. After the cooling with liquid hydrogen, he found on spectroscopic examination, in one no hydrogen, but two faint lines, one about 5852 W.L. and the other 5676 W.L. The second tube showed the same yellow about $5^{8} 5^{2}$, the helium line along with 5939 and 6145 , the hydrogen lines $\mathrm{C}$ and $\mathrm{F}$, and some red lines. The observations of Crookes confirm the presence of neon, helium, and hydrogen. The absence in his tubes of the carbonic oxide spectrum is important, seeing all the electrodeless tubes gave this spectrum. In these tubes the vacuum was very high, and it was difficult to observe the gaseous spectrum. Still, the fact of finding hydrogen in one and not in the other, leaves the presence of free hydrogen in the atmosphere as a question for further inquiry. The tube that did nut contain hydrogen was heated very hot in order to get a discharge, and then the spectrum showed some bands like the negative glow of nitrogen. Occasionally, a jar discharge was got to pass, and when this took place the nitrogen lines could be seen. An electrodeless tube filled carefully with oxygen made from fused chlorate of potash, which was contained in an extension of the vacuum tube, gave nothing but the carbonic oxide bands. In future experiments it will be easy to concentrate all the least volatile material in air or other gases, and thereby to make a more thorough examination of the spectrum. In the meantime my object is to show one of the scientific uses of liquid hydrogen.

I have to thank Mr. Robert Lennox for efficient aid in the conduct of the difficult experiments. Mr. Heath has also helped in the work.

1 In a paper along with Prof. Liveing, "On the Spectrum of the Electric Discharge in Liquid Oxygen, Air, and Nitrogen "(Phil. Mag., 1894), we noted that during the distillation and concentration in vacuo of liquid oxygen and air under diminished pressure, that two bright lines appeared in the spectrum at wave-length 557 and 555 , and that one of these lines wa very near the position of the auronal line. These lines are now attributed by the same chemists to a new element, crypton. 